\title{
Phosphorylation of alcohols with N-phosphoryl oxazolidinones employing copper (II) triflate catalysis
}

\author{
Simon Jones and Chaiwat Smanmoo \\ Department of Chemistry, University of Sheffield, Dainton Building, Brook Hill, \\ Sheffield S3 7HF, UK \\ E-mail: $\underline{\text { Simon.Jones@Sheffield.ac.uk }}$
}

\section{Supporting Information - Contents}

\section{Document A - Experimental}

General experimental procedures $\quad 2$

$N$-(5,5-Diphenyl oxazolidinyl) diethyl phosphate 7

$N$-(5,5-Diphenyl oxazolidinyl) diphenyl phosphate 8

Optimized procedure for phosphoryl transfer 4

Benzyl Diethyl Phosphate 9

Allyl Diethyl Phosphate 10 4

(Pro-2-ynyl) Diethyl Phosphate 11

Cyclohexyl Diethyl Phosphate 12

Isopropyl Diethyl Phosphate 13

2-Butyl Diethyl Phosphate 14

(2-Methyl-2-Propyl) Diethyl Phosphate 15

Terpineol Diethyl Phosphate $16 \quad 6$

(4-Bromophenyl) Diethyl Phosphate $17 \quad 6$

(4-Nitrophenyl) Diethyl Phosphate $18 \quad 6$

(4-Methoxyphenyl) Diethyl Phosphate $19 \quad 6$

$\begin{array}{ll}\text { Benzyl Diphenyl Phosphate } 20 & 7\end{array}$

$\begin{array}{ll}\text { Allyl Diphenyl Phosphate } 21 & 7\end{array}$

(Pro-2-ynyl) Diphenyl Phosphate 22 7

$\begin{array}{ll}\text { Cyclohexyl Diphenyl Phosphate 23 } & 7\end{array}$

$\begin{array}{ll}\text { Isopropyl Diphenyl Phosphate } 24 & 7\end{array}$

2-Butyl Diphenyl Phosphate 25

(2-Methyl-2-Propyl) Diphenyl Phosphate $26 \quad 8$ 
Terpineol Diphenyl Phosphate 27

(4-Bromophenyl) Diphenyl Phosphate $28 \quad 8$

(4-Nitrophenyl) Diphenyl Phosphate 29

(4-Methoxyphenyl) Diphenyl Phosphate $30 \quad 9$

Geraniol Diphenyl Phosphate 31

Adenosine 5' Phenyl Phosphate $32 \quad 9$

$\begin{array}{ll}\text { Guanosine 5' Phenyl Phosphate } 33 & 10\end{array}$

17 $\beta$-Hydroxy-5 $\alpha$-Androstan-3-one Diphenyl Phosphate $34 \quad 10$

$\begin{array}{ll}\text { References } & 10\end{array}$

\section{Document B - Copies of all ${ }^{1} \mathrm{H}$ nmr spectra}

\section{General Experimental Procedures.}

All solvents used were freshly dried over sodium (except dichloromethane which was dried over calcium hydride). Glassware was flame dried and cooled under vacuum before use. All reactions were carried out under the nitrogen atmosphere. TLC was carried out using aluminium backed tlc sheets (silica gel $60 \mathrm{~F}_{254}$ ) and visualization of the plates performed using a U.V. lamp or by dipping in $\mathrm{KMnO}_{4}$ then exposure by heating. For nmr experiments, residual proton signals from the deuteriated solvents were used as references [(chloroform (1H 7.25 ppm, 13C 77 ppm)]. Ligands 2 and 6 were purchased from commercial sources, while ligands $\mathbf{1}, \mathbf{3}, \mathbf{4}$, and $\mathbf{5}$ were prepared by existing literature methods. ${ }^{1}$

\section{$N$-(5,5-Diphenyl oxazolidinyl) diethyl phosphate $7^{2}$}

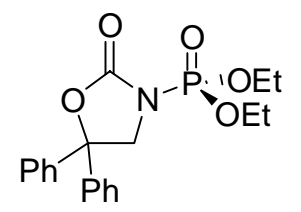

n-BuLi (1.68 mL, $4.20 \mathrm{mmol}, 2.5 \mathrm{M}$ solution in hexanes) was added to a stirred suspension of 5,5-diphenyl-oxazolidin-2-one ${ }^{3}(1.00 \mathrm{~g}, 4.20 \mathrm{mmol})$ at $-78{ }^{\circ} \mathrm{C}$ in THF $(15 \mathrm{~mL})$, and left to stir at RT for $1 \mathrm{~h}$. The solution was re-cooled to $-78{ }^{\circ} \mathrm{C}$, diethylchlorophosphate $(0.60 \mathrm{~mL}, 4.20 \mathrm{mmol})$ added dropwise and the solution left to stir at $\mathrm{RT}$ for $2 \mathrm{~h}$. Saturated $\mathrm{NH}_{4} \mathrm{Cl}$ solution $(10 \mathrm{~mL})$ was added and the mixture extracted with EtOAc $(3 \times 15 \mathrm{~mL})$. The combined organic extracts were washed with 
sat. $\mathrm{NaHCO}_{3}$ solution $(10 \mathrm{~mL})$ and brine $(10 \mathrm{~mL})$ and dried with $\mathrm{MgSO}_{4}$. Filtration, evaporation of solvent and purification by chromatography on silica gel (EtOAc: petroleum ether; 1:9) afforded the title compound as an oil (0.98 g, $63 \%)$; mpt. 70 $72{ }^{\circ} \mathrm{C} ; \mathrm{R}_{\mathrm{f}} 0.37$ (EtOAc: petroleum ether; 1:9); $R_{f} 0.37$ (EtOAc: petroleum ether; 15:85); IR (KBr) 2923, 2853, $1783(C=O), 1452 ;{ }^{1} \mathrm{H}$ NMR $\left(250 \mathrm{MHz}, \mathrm{CDCl}_{3}\right) \delta 1.26(6 \mathrm{H}, \mathrm{t}$, $J$ 6.5, $\left.\mathrm{CH}_{2} \mathrm{CH}_{3}\right), 4.07\left(4 \mathrm{H}, \mathrm{m}, \mathrm{CH}_{2} \mathrm{CH}_{3}\right), 4.50\left(2 \mathrm{H}, \mathrm{s}, \mathrm{CH}_{2}\right), 7.25-7.48(10 \mathrm{H}, \mathrm{m}$, $\mathrm{ArCH}) ;{ }^{13} \mathrm{C}$ NMR $\left(63 \mathrm{MHz}, \mathrm{CDCl}_{3}\right) \delta 15.9$ (d, $\left.J_{\mathrm{C}-\mathrm{P}} 6.8, \mathrm{CH}_{2} \mathrm{CH}_{3}\right), 57.1\left(\mathrm{~d}, J_{\mathrm{C}-\mathrm{P}} 3.7\right.$, $\left.\mathrm{CH}_{2} \mathrm{NH}\right), 64.6\left(\mathrm{~d}, J_{\mathrm{C}-\mathrm{P}} 5.9, C \mathrm{Ph}\right), 85.8\left(\mathrm{~d}, J_{\mathrm{C}-\mathrm{P}} 8.1, \mathrm{CH}_{2} \mathrm{O}\right), 124.5(\mathrm{ArCH}), 128.5$ $(\mathrm{ArCH}), 128.8(\mathrm{ArCH}), 141.2(\mathrm{ArC}), 154.1\left(\mathrm{~d}, J_{\mathrm{C}-\mathrm{P}} 7.7, C=\mathrm{O}\right) ;{ }^{31} \mathrm{P}$ NMR $(101 \mathrm{MHz}$, $\left.\mathrm{CDCl}_{3}\right) \delta-4.51$; MS (EI) $m / z 375\left(8 \%, \mathrm{M}^{+}\right), 331\left(\mathrm{M}^{+}-\mathrm{CO}_{2}, 58\right), 319(35), 298\left(\mathrm{M}^{+}-\mathrm{Ph}\right.$, 24), 183 (70), 178 (79), 165 (98), 138 (100), 105 (71); Anal. Calcd. for $\mathrm{C}_{19} \mathrm{H}_{22} \mathrm{NO}_{5} \mathrm{P}$ : C, 60.80; H 5.91; N 3.73. Found: C, 60.0; H, 5.72; N, 3.54.

\section{$N$-(5,5-Diphenyl oxazolidinyl) diphenyl phosphate $8^{2}$}

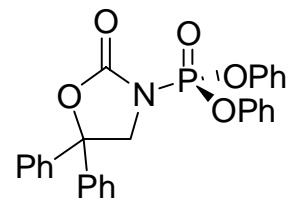

n-BuLi (1.68 mL, $4.20 \mathrm{mmol}, 2.5 \mathrm{M}$ solution in hexanes) was added to a stirred suspension of 5,5-diphenyl-oxazolidin-2-one ${ }^{3}(1.00 \mathrm{~g}, 4.20 \mathrm{mmol})$ at $-78^{\circ} \mathrm{C}$ in THF $(15 \mathrm{~mL})$, and left to stir at RT for $1 \mathrm{~h}$. The solution was re-cooled to $-78{ }^{\circ} \mathrm{C}$, diphenylchlorophosphate $(0.37 \mathrm{~mL}, 4.20 \mathrm{mmol})$ added dropwise and the solution left to stir at $\mathrm{RT}$ for $2 \mathrm{~h}$. Saturated $\mathrm{NH}_{4} \mathrm{Cl}$ solution $(10 \mathrm{~mL})$ was added and the mixture extracted with EtOAc $(3 \times 15 \mathrm{~mL})$. The combined organic extracts were washed with sat. $\mathrm{NaHCO}_{3}$ solution $(10 \mathrm{~mL})$ and brine $(10 \mathrm{~mL})$ and dried with $\mathrm{MgSO}_{4}$. Filtration, evaporation of solvent and purification by chromatography on silica gel (EtOAc: petroleum ether; 15:85) afforded the title compound as an oil (1.16 g, $74 \%) ; R_{f} 0.42$ (EtOAc: petroleum ether; 15:85); IR (KBr) 2925, $1775(C=O), 1589,1490,1458 ;{ }^{1} \mathrm{H}$ NMR (300 MHz, $\left.\mathrm{CDCl}_{3}\right) \delta 4.46\left(2 \mathrm{H}, \mathrm{s}, \mathrm{CH}_{2}\right), 6.95-7.38(20 \mathrm{H}, \mathrm{m}, \mathrm{ArCH}) ;{ }^{13} \mathrm{C} \mathrm{NMR}$ $\left(126 \mathrm{MHz}, \mathrm{CDCl}_{3}\right) \delta 57.3\left(\mathrm{~d}, J_{\mathrm{C}-\mathrm{P}} 3.6, \mathrm{CH}_{2} \mathrm{~N}\right), 86.1\left(\mathrm{~d}, J_{\mathrm{C}-\mathrm{P}} 8.9, C \mathrm{Ph}\right), 120.4\left(\mathrm{~d}, J_{\mathrm{C}-\mathrm{P}}\right.$ 4.7, $\mathrm{ArCH}), 125.3(\mathrm{ArCH}), 125.9(\mathrm{ArCH}), 128.5(\mathrm{ArCH}), 128.7(\mathrm{ArCH}), 129.8$ $(\mathrm{ArCH}), 140.7(\mathrm{ArC}), 149.5\left(\mathrm{~d}, J_{\mathrm{C}-\mathrm{P}} 6.7, \mathrm{ArC}\right), 153.4\left(\mathrm{~d}, J_{\mathrm{C}-\mathrm{P}} 7.5, C=\mathrm{O}\right) ;{ }^{31} \mathrm{P}$ NMR $\left(101 \mathrm{MHz}, \mathrm{CDCl}_{3}\right) \delta$-12.79; MS (TOF ES) $m / z 471\left(100 \%, \mathrm{M}^{+}\right)$; Anal. Calcd. for $\mathrm{C}_{17} \mathrm{H}_{18} \mathrm{NO}_{5} \mathrm{P}: \mathrm{C}, 68.79 ; \mathrm{H}, 4.70 ; \mathrm{N}, 2.97$. Found: C, 69.34; H, 5.10; N, 2.72. 


\section{Optimized procedure for phosphoryl transfer}

Alcohol (1.0 mmol) was added to a stirred solution of copper (II) triflate $(0.2 \mathrm{mmol})$ and $N, N^{\prime}$-ethylenebis(benzaldimine) $(\mathrm{BEN})(0.2 \mathrm{mmol})$ in $\mathrm{CH}_{2} \mathrm{Cl}_{2}(10 \mathrm{~mL})$ at $\mathrm{RT}$ under a nitrogen atmosphere. The solution was left stirring at RT for $5 \mathrm{~min}$ before $\mathrm{Et}_{3} \mathrm{~N}$ ( $\left.1 \mathrm{mmol}\right)$ and dialkyl $\mathrm{N}$-phosphoryl oxazolidinone $(1 \mathrm{mmol})$ were added sequentially. The reaction mixture was left to stir at RT for $48 \mathrm{~h}$ before adding $\mathrm{NH}_{4} \mathrm{Cl}_{(\mathrm{aq})}(10 \mathrm{~mL})$, and extracting with EtOAc $(3 \times 30 \mathrm{~mL})$. The combined organic layers were washed with $\mathrm{NaHCO}_{3}(50 \mathrm{~mL})$ and brine $(50 \mathrm{~mL})$ and dried over $\mathrm{MgSO}_{4}$. The suspension was filtered and the solvent was removed under reduced pressure. The phosphate product was obtained after purification by appropriate silica gel column chromatography (ethyl acetate / petroleum ether).

\section{Benzyl Diethyl Phosphate $9^{4}$}<smiles>CCOP(=O)(OCC)OCc1ccccc1</smiles>

Obtained in $86 \%$ yield as clear oil; ${ }^{1} \mathrm{H}$ NMR $\left(250 \mathrm{MHz}, \mathrm{CDCl}_{3}\right) \delta 1.18(6 \mathrm{H}, \mathrm{t}, J 6.8$, $\mathrm{CH}_{3} \mathrm{CH}_{2}$ ), 3.88 (4H, app pent, $J_{\mathrm{P}-\mathrm{H}} 6.8$ and $\left.J 6.8, \mathrm{CH}_{2} \mathrm{CH}_{3}\right), 4.87\left(2 \mathrm{H}, \mathrm{d}, J_{\mathrm{P}-\mathrm{H}} 8.5\right.$, $\left.\mathrm{CH}_{2} \mathrm{O}\right), 7.10-7.22(5 \mathrm{H}, \mathrm{m}, \mathrm{ArCH}) ;{ }^{31} \mathrm{P} \mathrm{NMR}\left(101 \mathrm{MHz}, \mathrm{CDCl}_{3}\right) \delta-0.58$.

\section{Allyl Diethyl Phosphate $10^{5}$}

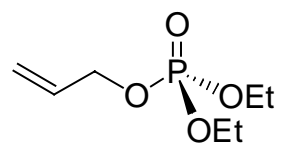

Obtained in $84 \%$ yield as a colorless oil; ${ }^{1} \mathrm{H}$ NMR $\left(250 \mathrm{MHz}, \mathrm{CDCl}_{3}\right) \delta 1.29(6 \mathrm{H}$, td, $J 7.1$ and $\left.J_{\mathrm{P}-\mathrm{H}} 1.1, \mathrm{CH}_{3} \mathrm{CH}_{2}\right), 4.06\left(4 \mathrm{H}\right.$, app pent, $J_{\mathrm{P}-\mathrm{H}} 7.1$ and $\left.J 7.1, \mathrm{CH}_{2} \mathrm{CH}_{3}\right), 4.47$ $\left(2 \mathrm{H}, \mathrm{m}, \mathrm{CH}_{2} \mathrm{OP}\right), 5.19\left(1 \mathrm{H}, \mathrm{dq}, J 17.1\right.$ and $\left.1.3, \mathrm{CH}=\mathrm{CH}_{2}\right), 5.31(1 \mathrm{H}, \mathrm{dq}, J 10.4$ and 1.3, $\left.\mathrm{CH}=\mathrm{CH}_{2}\right), 5.89\left(1 \mathrm{H}, \mathrm{m}, \mathrm{CH}=\mathrm{CH}_{2}\right) ;{ }^{31} \mathrm{P}\left(101 \mathrm{MHz}, \mathrm{CDCl}_{3}\right) \delta-0.57$.

(Pro-2-ynyl) Diethyl Phosphate $11^{6}$

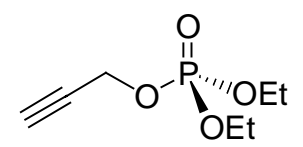

Obtained in 75\% yield as a colorless oil; ${ }^{1} \mathrm{H}$ NMR $\left(250 \mathrm{MHz}, \mathrm{CDCl}_{3}\right) \delta 1.39(6 \mathrm{H}$, td, $J 7.0$ and $\left.J_{\mathrm{P}-\mathrm{H}} 1.2, \mathrm{CH}_{2} \mathrm{CH}_{3}\right), 2.50(1 \mathrm{H}, \mathrm{t}, J 2.5, \mathrm{C} \equiv \mathrm{CH}), 4.06\left(2 \mathrm{H}, \mathrm{d}, J 2.5, \mathrm{OCH}_{2}\right)$, $4.21\left(4 \mathrm{H}, \mathrm{m}, \mathrm{CH}_{2} \mathrm{CH}_{3}\right) ;{ }^{31} \mathrm{P} \mathrm{NMR}\left(101 \mathrm{MHz}, \mathrm{CDCl}_{3}\right) \delta-0.69$. 


\section{Cyclohexyl Diethyl Phosphate $12^{7}$}<smiles>CCOP(=O)(OCC)OC1CCCCC1</smiles>

Obtained in $80 \%$ yield as clear oil; ${ }^{1} \mathrm{H}$ NMR $\left(300 \mathrm{MHz}, \mathrm{CDCl}_{3}\right) \delta 1.16-1.27(3 \mathrm{H}, \mathrm{m}$, $\left.\mathrm{CH}_{2}\right), 1.25\left(6 \mathrm{H}\right.$, td, $J$ 7.1, and $\left.J_{\mathrm{P}-\mathrm{H}} 1.0, \mathrm{CH}_{3} \mathrm{CH}_{2}\right), 1.32-1.49\left(3 \mathrm{H}, \mathrm{m}, \mathrm{CH}_{2}\right), 1.58-1.69$ $\left(2 \mathrm{H}, \mathrm{m}, \mathrm{CH}_{2}\right), 1.76-1.88\left(2 \mathrm{H}, \mathrm{m}, \mathrm{CH}_{2}\right), 3.96$ (4H, app pent, $J_{\mathrm{P}-\mathrm{H}} 7.1$ and $J$ 7.1, $\left.\mathrm{CH}_{2} \mathrm{CH}_{3}\right), 4.24(1 \mathrm{H}, \mathrm{m}, \mathrm{CHOP}) ;{ }^{31} \mathrm{P}$ NMR (101 MHz, $\left.\mathrm{CDCl}_{3}\right) \delta-1.67$.

\section{Isopropyl Diethyl Phosphate $13^{8}$}

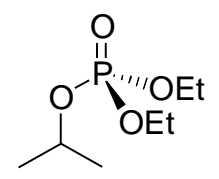

Obtained in $80 \%$ yield as a clear oil; ${ }^{1} \mathrm{H}$ NMR $\left(250 \mathrm{MHz}, \mathrm{CDCl}_{3}\right) \delta 1.25-1.31(12 \mathrm{H}$, m, $\mathrm{CH}_{3} \mathrm{CH}_{2}$ and $\left.\mathrm{CH}_{3} \mathrm{CH}\right), 4.03$ (4H, app pent, $J_{\mathrm{P}-\mathrm{H}} 7.3$ and $\left.J 7.3, \mathrm{CH}_{2} \mathrm{CH}_{3}\right), 4.55(1 \mathrm{H}$, $\mathrm{m}, \mathrm{CHOP}) ;{ }^{31} \mathrm{P}\left(101 \mathrm{MHz}, \mathrm{CDCl}_{3}\right) \delta-1.48$.

\section{2-Butyl Diethyl Phosphate $14^{9}$}

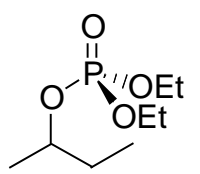

Obtained in $77 \%$ yield as a clear oil; ${ }^{1} \mathrm{HNMR}\left(250 \mathrm{MHz}, \mathrm{CDCl}_{3}\right) \delta 0.80(3 \mathrm{H}, \mathrm{t}, J 7.5$, $\left.\mathrm{CH}_{3} \mathrm{CH}_{2}\right), 1.21\left(6 \mathrm{H}, \mathrm{td}, J 7.2\right.$ and $\left.J_{\mathrm{P}-\mathrm{H}} 0.8, \mathrm{CH}_{2} \mathrm{CH}_{3}\right), 1.41-1.62\left(3 \mathrm{H}, \mathrm{m}, \mathrm{CH}_{2} \mathrm{CH}\right)$, $3.95\left(4 \mathrm{H}, \mathrm{m}, \mathrm{CH}_{2} \mathrm{CH}_{3}\right), 4.29\left(1 \mathrm{H}\right.$, app hept, $J_{\mathrm{P}-\mathrm{H}} 6.3$ and $\left.J 6.3, \mathrm{CHOP}\right) ;{ }^{31} \mathrm{P}(101 \mathrm{MHz}$, $\left.\mathrm{CDCl}_{3}\right) \delta-1.35$.

(2-Methyl-2-Propyl) Diethyl Phosphate $15^{10}$

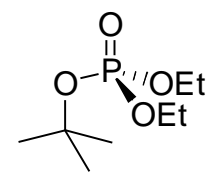

Obtained in 55\% yield as a clear oil; ${ }^{1} \mathrm{H}$ NMR $\left(250 \mathrm{MHz}, \mathrm{CDCl}_{3}\right) \delta 1.03(6 \mathrm{H}$, td, $J 6.5$ and $\left.J_{\mathrm{P}-\mathrm{H}} 0.9, \mathrm{CH}_{2} \mathrm{CH}_{3}\right), 1.18\left(9 \mathrm{H}, \mathrm{s}, \mathrm{CH}_{3} \mathrm{C}\right), 3.74\left(4 \mathrm{H}\right.$, app pent, $J_{\mathrm{P}-\mathrm{H}} 6.5$ and $J 6.5$, $\left.\mathrm{CH}_{2} \mathrm{CH}_{3}\right) ;{ }^{31} \mathrm{P}$ NMR (101 MHz, $\left.\mathrm{CDCl}_{3}\right) \delta-6.98$. 
( \pm )- $\alpha$-Terpineol Diethyl Phosphate $16^{2}$

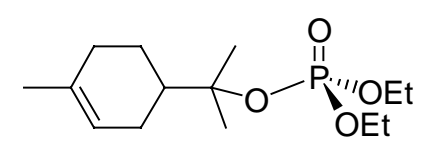

Obtained in 55\% yield as a clear oil; IR / $\mathrm{cm}^{-1}$ (ATR) 2966, 2922, 2889, 1438, 1376, 1384, 1154; ${ }^{1} \mathrm{H}$ NMR $\left(250 \mathrm{MHz}, \mathrm{CDCl}_{3}\right) \delta 1.16\left(3 \mathrm{H}, \mathrm{s}, \mathrm{CH}_{3}\right), 1.20\left(3 \mathrm{H}, \mathrm{s}, \mathrm{CH}_{3}\right)$, $1.21-1.56(2 \mathrm{H}, \mathrm{m}), 1.40\left(6 \mathrm{H}, \mathrm{td}, J 6.9\right.$ and $\left.J_{\mathrm{P}-\mathrm{H}} 1.2, \mathrm{CH}_{3} \mathrm{CH}_{2}\right), 1.63\left(3 \mathrm{H}, \mathrm{s}, \mathrm{CH}_{3}\right), 1.70$ $2.13(5 \mathrm{H}, \mathrm{m}), 4.28\left(4 \mathrm{H}\right.$, app pent, $J 6.9$ and $\left.J_{\mathrm{P}-\mathrm{H}} 6.9, \mathrm{CH}_{2} \mathrm{CH}_{3}\right), 5.34(1 \mathrm{H}, \mathrm{s}, \mathrm{CH}=\mathrm{C})$; ${ }^{13} \mathrm{C}$ NMR $\left(75 \mathrm{MHz}, \mathrm{CDCl}_{3}\right) \delta 15.6\left(\mathrm{~d}, J_{\mathrm{C}-\mathrm{P}} 7.8, \mathrm{CH}_{3}\right), 23.3\left(\mathrm{CH}_{3}\right), 23.9\left(\mathrm{CH}_{2}\right), 26.2$ $\left(\mathrm{CH}_{3}\right), 26.9\left(\mathrm{CH}_{2}\right), 27.4\left(\mathrm{CH}_{3}\right), 31.0\left(\mathrm{CH}_{2}\right), 44.9(\mathrm{CH}), 65.7\left(\mathrm{~d}, J_{\mathrm{C}-\mathrm{P}} 6.8, \mathrm{CH}_{2} \mathrm{O}\right), 72.7$ $\left[\left(\mathrm{CH}_{3}\right)_{2} \mathrm{CO}\right], 120.5(\mathrm{CH}=\mathrm{C}), 133.9(\mathrm{CH}=\mathrm{C}) ;{ }^{31} \mathrm{P} \mathrm{NMR}\left(101 \mathrm{MHz}, \mathrm{CDCl}_{3}\right) \delta-5.16 ; \mathrm{m} / z$ (EI) 291 (58\%, MH ), 263 (52), 235 (47), 207 (42), 179 (62), 161 (100), 99 (83), 68 (70); Anal. Calcd for $\mathrm{C}_{14} \mathrm{H}_{27} \mathrm{O}_{4} \mathrm{P}: \mathrm{C}, 57.92 ; \mathrm{H}$ 9.37. Found: C, 58.20; H, 9.44.

(4-Bromophenyl) Diethyl Phosphate $17^{11}$

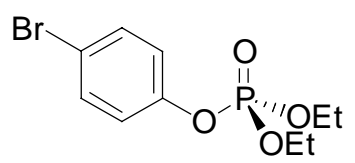

Obtained in 54\% yield as a white solid; ${ }^{1} \mathrm{H}$ NMR $\left(250 \mathrm{MHz}, \mathrm{CDCl}_{3}\right) \delta 1.27(6 \mathrm{H}, \mathrm{td}, J$ 6.9 and $\left.J_{\mathrm{P}-\mathrm{H}} 1.1, \mathrm{CH}_{2} \mathrm{CH}_{3}\right), 4.15$ (4H, app pent, $J_{\mathrm{P}-\mathrm{H}} 6.9$ and $\left.J 6.9, \mathrm{CH}_{2} \mathrm{CH}_{3}\right), 6.98(2 \mathrm{H}$, d, $J$ 8.7, $\mathrm{ArCH}), 7.31(2 \mathrm{H}, \mathrm{d}, J$ 8.7, $\mathrm{ArCH}) ;{ }^{31} \mathrm{P} \mathrm{NMR}\left(101 \mathrm{MHz}, \mathrm{CDCl}_{3}\right) \delta-6.16$.

\section{(4-Nitrophenyl) Diethyl Phosphate $18^{12}$}

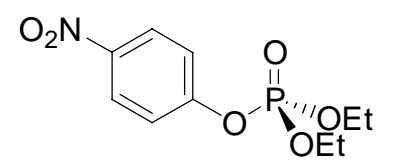

Obtained in $51 \%$ yield as a clear oil; ${ }^{1} \mathrm{H}$ NMR $\left(250 \mathrm{MHz}, \mathrm{CDCl}_{3}\right) \delta 1.26(6 \mathrm{H}, \mathrm{t}, J$ J.2, $\mathrm{CH}_{2} \mathrm{CH}_{3}$ ), 4.15 (4H, app pent, $J_{\mathrm{P}-\mathrm{H}} 7.2$ and J 7.2, $\left.\mathrm{CH}_{2} \mathrm{CH}_{3}\right), 7.29(2 \mathrm{H}, \mathrm{d}, J$ 9.2, $\mathrm{ArCH}$ ), $8.15(2 \mathrm{H}, \mathrm{d}, J 9.2, \mathrm{ArCH}) ;{ }^{31} \mathrm{P} \mathrm{NMR}\left(101 \mathrm{MHz}, \mathrm{CDCl}_{3}\right) \delta-7.36$.

\section{(4-Methoxyphenyl) Diethyl Phosphate $19^{13}$}

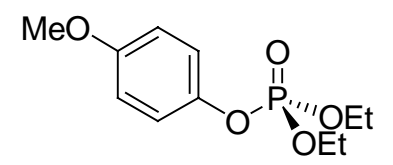

Obtained in $31 \%$ yield as a clear oil; ${ }^{1} \mathrm{H}$ NMR $\left(250 \mathrm{MHz}, \mathrm{CDCl}_{3}\right) \delta 1.23(6 \mathrm{H}, \mathrm{td}, J 6.2$ and $\left.J_{\mathrm{P}-\mathrm{H}} 0.9, \mathrm{CH}_{2} \mathrm{CH}_{3}\right), 3.65\left(3 \mathrm{H}, \mathrm{d}, J_{\mathrm{P}-\mathrm{H}} 0.9, \mathrm{OCH}_{3}\right), 4.10\left(4 \mathrm{H}\right.$, app pent, $J_{\mathrm{P}-\mathrm{H}} 6.4$ and $\left.J 6.4, \mathrm{CH}_{2} \mathrm{CH}_{3}\right), 6.72\left(2 \mathrm{H}, \mathrm{dd}, J 9.7\right.$ and $\left.J_{\mathrm{P}-\mathrm{H}} 1.5, \mathrm{ArCH}\right), 7.03\left(2 \mathrm{H}, \mathrm{dd}, J 9.7\right.$ and $J_{\mathrm{P}-\mathrm{H}}$ 1.5, $\mathrm{ArCH}) ;{ }^{31} \mathrm{P}$ NMR $\left(101 \mathrm{MHz}, \mathrm{CDCl}_{3}\right) \delta-5.54$. 


\section{Benzyl Diphenyl Phosphate 20}<smiles>O=P(OCc1ccccc1)(Oc1ccccc1)Oc1ccccc1</smiles>

Obtained in $85 \%$ yield as white solid; $\delta_{\mathrm{H}}\left(250 \mathrm{MHz} ; \mathrm{CDCl}_{3}\right) 5.27(2 \mathrm{H}, \mathrm{d}, J 8.5$, $\left.\mathrm{CH}_{2} \mathrm{OP}\right), 7.14-7.21(6 \mathrm{H}, \mathrm{m}, \mathrm{ArCH}), 7.30-7.38(9 \mathrm{H}, \mathrm{m}, \mathrm{ArCH}) ; \delta_{\mathrm{P}}\left(101 \mathrm{MHz} ; \mathrm{CDCl}_{3}\right)$ -11.31 .

Allyl Diphenyl Phosphate $21^{7}$

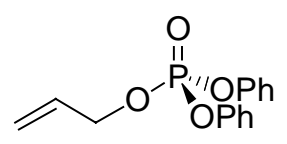

Obtained in $88 \%$ yield colorless oil; $\delta_{\mathrm{H}}\left(250 \mathrm{MHz} ; \mathrm{CDCl}_{3}\right) 4.66\left(2 \mathrm{H}\right.$, ddt, $J_{\mathrm{P}-\mathrm{H}} 8.6, J$ 5.7 and 1.4, $\left.\mathrm{CH}_{2} \mathrm{O}\right), 5.17\left(1 \mathrm{H}, \mathrm{dd}, J 10.4\right.$ and $\left.1.2, \mathrm{CH}=\mathrm{CH}_{2}\right), 5.28(\mathrm{H}, \mathrm{dq}, J 17.0$ and 1.2, $\left.\mathrm{CH}=\mathrm{CH}_{2}\right), 5.86\left(1 \mathrm{H}, \mathrm{m}, \mathrm{CH}=\mathrm{CH}_{2}\right), 6.83-7.31(10 \mathrm{H}, \mathrm{m}, \mathrm{ArCH}) ; \delta_{\mathrm{P}}(101 \mathrm{MHz}$; $\left.\mathrm{CDCl}_{3}\right)-11.60$.

(Pro-2-ynyl) Diphenyl Phosphate 22 ${ }^{7}$

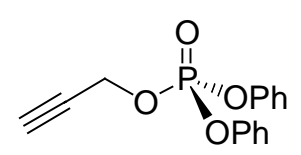

Obtained in $84 \%$ yield as read-brown oil; $\delta_{\mathrm{H}}\left(250 \mathrm{MHz} ; \mathrm{CDCl}_{3}\right) 2.51(1 \mathrm{H}, \mathrm{t}, J$ 2.6, $\mathrm{CH}), 4.06\left(2 \mathrm{H}, \mathrm{d}, J 2.6, \mathrm{CH}_{2} \mathrm{O}\right), 7.25-7.45(10 \mathrm{H}, \mathrm{m}, \mathrm{ArCH}) ; \delta_{\mathrm{P}}\left(101 \mathrm{MHz} ; \mathrm{CDCl}_{3}\right)-$ 11.5 .

Cyclohexyl Diphenyl Phosphate $23^{7}$

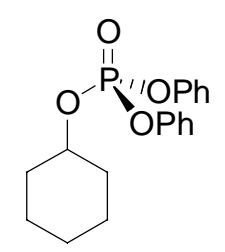

Obtained in $82 \%$ yield as clear oil; $\delta_{\mathrm{H}}\left(250 \mathrm{MHz} ; \mathrm{CDCl}_{3}\right) 1.21-1.40\left(3 \mathrm{H}, \mathrm{m}, \mathrm{CH}_{2}\right)$, 1.42-1.81 (5H, m, CH $), 1.85-2.00\left(2 \mathrm{H}, \mathrm{m}, \mathrm{CH}_{2}\right), 4.62(1 \mathrm{H}, \mathrm{m}, \mathrm{CHO}), 7.15-7.39$ $(10 \mathrm{H}, \mathrm{m}, \mathrm{ArCH}) ; \delta_{\mathrm{P}}\left(101 \mathrm{MHz} ; \mathrm{CDCl}_{3}\right)-12.28$.

\section{Isopropyl Diphenyl Phosphate $24^{7}$}<smiles>CC(C)OP(=O)(Oc1ccccc1)c1ccccc1</smiles> 
Obtained in $85 \%$ yield as clear oil; $\delta_{\mathrm{H}}\left(250 \mathrm{MHz} ; \mathrm{CDCl}_{3}\right) 1.23\left(6 \mathrm{H}, \mathrm{d}, J 6.3, \mathrm{CH}_{3} \mathrm{CH}\right)$, $4.77\left(1 \mathrm{H}\right.$, app hex $J 6.3$ and $\left.J_{\mathrm{P}-\mathrm{H}} 6.3, \mathrm{CHO}\right), 7.20-7.24(10 \mathrm{H}, \mathrm{m}, \mathrm{ArCH}) ; \delta_{\mathrm{P}}(101 \mathrm{MHz}$; $\left.\mathrm{CDCl}_{3}\right)-12.43$.

\section{2-Butyl Diphenyl Phosphate $25^{14}$}

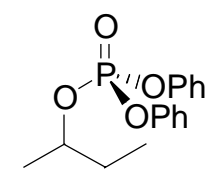

Obtained in $75 \%$ yield as clear oil; $\delta_{\mathrm{H}}\left(250 \mathrm{MHz} ; \mathrm{CDCl}_{3}\right) 0.76\left(3 \mathrm{H}, \mathrm{t}, J 6.0, \mathrm{CH}_{3} \mathrm{CH}_{2}\right)$, $1.19\left(3 \mathrm{H}, \mathrm{d}, J 6.1, \mathrm{CH}_{3} \mathrm{CH}\right), 1.40-1.59\left(2 \mathrm{H}, \mathrm{m}, \mathrm{CH}_{2} \mathrm{CH}_{3}\right), 4.57$ (1H, app hept $J 6.1$ and $\left.J_{\mathrm{P}-\mathrm{H}} 6.1, \mathrm{CHO}\right), 6.95-7.21(10 \mathrm{H}, \mathrm{m}, \mathrm{ArCH}) ; \delta_{\mathrm{P}}\left(101 \mathrm{MHz} ; \mathrm{CDCl}_{3}\right)-12.50$.

(2-Methyl-2-Propyl) Diphenyl Phosphate $26^{14}$

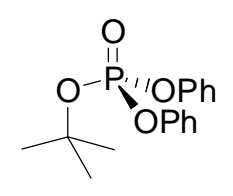

Obtained in $61 \%$ yield as clear oil; $\delta_{\mathrm{H}}\left(250 \mathrm{MHz} ; \mathrm{CDCl}_{3}\right) 1.52\left(9 \mathrm{H}, \mathrm{s}, \mathrm{CH}_{3} \mathrm{C}\right), 7.01-$ $7.33(10 \mathrm{H}, \mathrm{m}, \mathrm{ArCH}) ; \delta_{\mathrm{P}}\left(101 \mathrm{MHz} ; \mathrm{CDCl}_{3}\right)-10.26$.

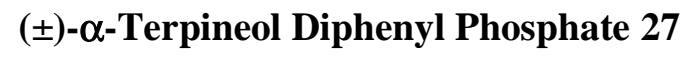

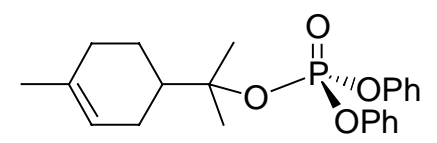

Obtained in $67 \%$ yield as clear oil; IR $(\mathrm{KBr}) 1488 ; \delta_{\mathrm{H}}\left(250 \mathrm{MHz} ; \mathrm{CDCl}_{3}\right) 1.14(3 \mathrm{H}, \mathrm{s}$, $\left.\mathrm{CH}_{3}\right), 1.18\left(3 \mathrm{H}, \mathrm{s}, \mathrm{CH}_{3}\right), 1.20-1.35(1 \mathrm{H}, \mathrm{m}), 1.37(1 \mathrm{H}, \mathrm{m}), 1.50(1 \mathrm{H}, \mathrm{m}), 1.65(3 \mathrm{H}, \mathrm{m}$, $\left.\mathrm{CH}_{3}\right), 1.70-2.13(4 \mathrm{H}, \mathrm{m}), 5.39(1 \mathrm{H}, \mathrm{m}, \mathrm{C}=\mathrm{CH}), 7.25-7.46(10 \mathrm{H}, \mathrm{m}, \mathrm{ArCH}) ; \delta_{\mathrm{C}}(63$ $\left.\mathrm{MHz} ; \mathrm{CDCl}_{3}\right) 23.4\left(\mathrm{CH}_{3}\right), 24.0\left(\mathrm{CH}_{2}\right), 26.2\left(\mathrm{CH}_{3}\right), 26.9\left(\mathrm{CH}_{2}\right), 27.4\left(\mathrm{CH}_{3}\right), 31.0$ $\left(\mathrm{CH}_{2}\right), 45.0(\mathrm{CH}), 72.8\left[\left(\mathrm{CH}_{3}\right)_{2} \mathrm{CO}\right], 120.4\left(\mathrm{~d}, J_{\mathrm{C}-\mathrm{P}} 5.1, \mathrm{ArCH}\right), 120.6(\mathrm{CH}=\mathrm{C}), 126.5$ $(\mathrm{ArCH}), 130.1\left(\mathrm{~d}, J_{\mathrm{C}-\mathrm{P}} 6.8, \operatorname{ArCH}\right), 134.0(\mathrm{CH}=C), 149.7\left(\mathrm{~d}, J_{\mathrm{C}-\mathrm{P}} 9.1, \operatorname{ArC}\right) ; \delta_{\mathrm{P}}(101$ $\mathrm{MHz} ; \mathrm{CDCl}_{3}$ ) -10.37; m/z (EI) $386\left(75 \%, \mathrm{M}^{+}\right), 216$ (50), 118 (55), 103 (100), 77 (40), 69 (30), 91 (28), 51 (25); Anal. Calcd. for $\mathrm{C}_{22} \mathrm{H}_{24} \mathrm{O}_{7} \mathrm{P}: \mathrm{C}, 68.38 ; \mathrm{H}$ 7.04. Found: C, $68.44 ; \mathrm{H}, 7.34$.

\section{(4-Bromophenyl) Diphenyl Phosphate $28^{15}$}

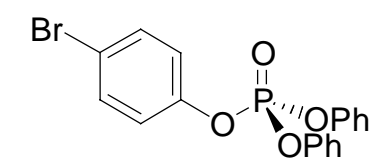


Obtained in $49 \%$ yield as clear oil; $\delta_{\mathrm{H}}\left(250 \mathrm{MHz} ; \mathrm{CDCl}_{3}\right) 7.14(2 \mathrm{H}, \mathrm{d}, J$ 8.7, $\mathrm{ArCH})$, 7.17-7.25 (6H, m, ArCH), 7.30-7.40 (4H, m, ArCH), 7.46 (2H, d, J 8.7, ArCH); $\delta_{\mathrm{P}}$ $\left(101 \mathrm{MHz} ; \mathrm{CDCl}_{3}\right)-16.73$.

(4-Nitrophenyl) Diphenyl Phosphate 29

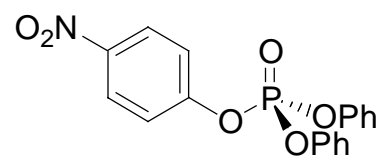

Obtained in $56 \%$ yield as clear oil; $\delta_{\mathrm{H}}\left(250 \mathrm{MHz} ; \mathrm{CDCl}_{3}\right) 7.15-7.42(12 \mathrm{H}, \mathrm{m}, \operatorname{ArCH})$, $8.20(2 \mathrm{H}, \mathrm{d}, J 8.9, \mathrm{ArCH}) ; \delta_{\mathrm{P}}\left(101 \mathrm{MHz} ; \mathrm{CDCl}_{3}\right)-18.07$.

(4-Methoxyphenyl) Diphenyl Phosphate 30 ${ }^{16}$

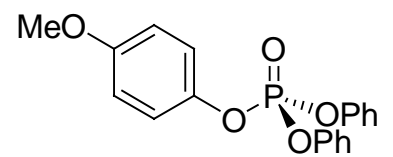

Obtained in $46 \%$ yield as clear oil; $\delta_{\mathrm{H}}\left(250 \mathrm{MHz}, \mathrm{CDCl}_{3}\right) 3.78\left(3 \mathrm{H}, \mathrm{s}, \mathrm{OCH}_{3}\right), 6.85$ (2H, d, J 8.6, $\mathrm{ArCH}), 7.13-7.38(12 \mathrm{H}, \mathrm{m}, \mathrm{ArCH}) ; \delta_{\mathrm{P}}\left(101 \mathrm{MHz} ; \mathrm{CDCl}_{3}\right)-11.49$.

\section{Geraniol Diphenyl Phosphate 31}

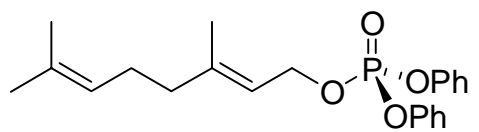

Obtained in $92 \%$ yield as clear oil; $\delta_{\mathrm{H}}\left(250 \mathrm{MHz} ; \mathrm{CDCl}_{3}\right) 1.50\left(3 \mathrm{H}, \mathrm{s}, \mathrm{CH}_{3}\right), 1.56(6 \mathrm{H}$, s, $\left.2 \times \mathrm{CH}_{3}\right), 1.82-2.02\left(4 \mathrm{H}, \mathrm{m}, 2 \times \mathrm{CH}_{2}\right), 4.63\left(1 \mathrm{H}, \mathrm{dd}, J_{\mathrm{P}-\mathrm{H}} 9.0\right.$ and $\left.J 7.5, \mathrm{CH}_{2} \mathrm{O}\right)$, $4.96(1 \mathrm{H}, \mathrm{m}, \mathrm{C}=\mathrm{CH}), 5.27(1 \mathrm{H}, \mathrm{td}, J 7.2$ and 1.2, $\mathrm{C}=\mathrm{CH}), 6.95-7.26(10 \mathrm{H}, \mathrm{m}, \mathrm{ArCH})$; $\delta_{\mathrm{P}}\left(101 \mathrm{MHz} ; \mathrm{CDCl}_{3}\right)-11.06$.

\section{Adenosine 5' Phenyl Phosphate $32^{17}$}

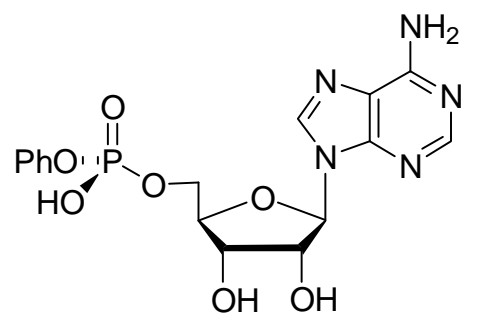

Obtained in $49 \%$ yield as white solid; mpt. $242{ }^{\circ} \mathrm{C} ;[\alpha]_{\mathrm{D}}-49.5\left(c 2, \mathrm{H}_{2} \mathrm{O}\right) ; \delta_{\mathrm{H}}(250$ $\left.\mathrm{MHz} ; \mathrm{D}_{2} \mathrm{O}\right) 3.92-4.10(2 \mathrm{H}, \mathrm{m}), 4.23(1 \mathrm{H}, \mathrm{m}), 4.36(1 \mathrm{H}, \mathrm{t}, J 5.0), 4.50(1 \mathrm{H}, \mathrm{t}, J 5.0)$, $5.83(1 \mathrm{H}, \mathrm{d}, J 5.0, \mathrm{H} 1$ ') $, 6.60(3 \mathrm{H}, \mathrm{m}, \mathrm{ArCH}), 7.04(2 \mathrm{H}, \mathrm{dd}, J 8.2$ and 7.6, $\operatorname{ArCH})$, $7.80(1 \mathrm{H}, \mathrm{s}, \operatorname{ArCH}), 8.23(1 \mathrm{H}, \mathrm{s}, \mathrm{ArCH}) ; \delta_{\mathrm{C}}\left(63 \mathrm{MHz} ; \mathrm{D}_{2} \mathrm{O}\right) 64.2(\mathrm{CH}), 70.1(\mathrm{CH})$, $74.4(\mathrm{CH}), 83.6\left(\mathrm{~d}, J_{\mathrm{C}-\mathrm{P}} 8.7, \mathrm{CH}_{2} \mathrm{O}\right), 87.2(\mathrm{CH}), 115.0(\mathrm{ArCH}), 117.9(\mathrm{ArC}), 120.3$ 
$(\mathrm{ArCH}), 129.6(\mathrm{ArCH}), 139.9(\mathrm{ArCH}), 148.1(\mathrm{ArC}), 150.7(\mathrm{ArCH}), 153.7(\mathrm{ArC})$, $155.1(\operatorname{ArC}) ; \delta_{\mathrm{P}}\left(101 \mathrm{MHz} ; \mathrm{D}_{2} \mathrm{O}\right) 2.48 ; \mathrm{m} / z$ (TOF ES) $423\left(\mathrm{M}^{+}\right)$.

\section{Guanosine 5' Phenyl Phosphate 33}

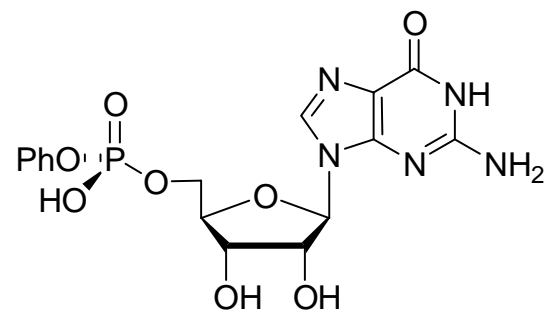

Obtained in 54\% yield as white solid; mpt. $256-257{ }^{\circ} \mathrm{C} ;[\alpha]_{\mathrm{D}}-86.4\left(c 2, \mathrm{H}_{2} \mathrm{O}\right) ; v_{\max } /$ $\mathrm{cm}^{-1}(\mathrm{KBr}) 2467,2362,1570,1540,1200 ; \delta_{\mathrm{H}}\left(250 \mathrm{MHz} ; \mathrm{D}_{2} \mathrm{O}\right) 3.91-3.99(2 \mathrm{H}, \mathrm{m})$, $4.25(1 \mathrm{H}, \mathrm{m}), 4.40$ (1H, app t J 5.0), 4.62 (1H, t, J 5.5), 5.82 (1H, d, J 5.5, H1'), 6.59$6.65(4 \mathrm{H}, \mathrm{m}, \mathrm{ArCH}), 7.08(2 \mathrm{H}$, app t, J 7.6, $\mathrm{ArCH}), 8.05(1 \mathrm{H}, \mathrm{s}, \mathrm{ArCH}) ; \delta_{\mathrm{C}}(63 \mathrm{MHz}$; $\left.\mathrm{D}_{2} \mathrm{O}\right) 63.4(\mathrm{CH}), 70.5(\mathrm{CH}), 74.0(\mathrm{CH}), 83.6\left(\mathrm{~d}, J_{\mathrm{C}-\mathrm{P}} 8.6, \mathrm{CH}_{2} \mathrm{O}\right), 86.4(\mathrm{CH}), 116.5$ $(\mathrm{ArCH}), 116.7(\mathrm{ArC}), 118.1(\mathrm{ArCH}), 129.7(\mathrm{ArCH}), 136.3(\mathrm{ArCH}), 151.4(\mathrm{ArC})$, 157.9 (ArC), $159.3(\operatorname{ArC}), 164.0(\operatorname{ArC}) ; \delta_{\mathrm{P}}\left(101 \mathrm{MHz} ; \mathrm{D}_{2} \mathrm{O}\right) 6.82 ; \mathrm{m} / z$ (TOF ES) 440 (100\%, $\mathrm{MH}^{+}$), 456 (35); Anal. Calcd. for $\mathrm{C}_{16} \mathrm{H}_{18} \mathrm{~N}_{5} \mathrm{O}_{8} \mathrm{P}: \mathrm{C}, 43.74 ; \mathrm{H} 4.13 ; \mathrm{N} 15.94$. Found: C, 43.8; H, 4.2; N, 16.0.

\section{7 $\beta$-Hydroxy-5 $\alpha$-Androstan-3-one Diphenyl Phosphate $34^{7}$}

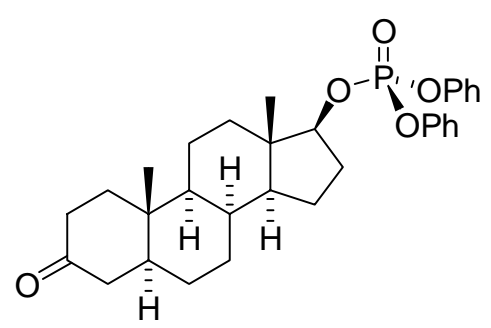

Obtained in $76 \%$ yield as a white solid; $\delta_{\mathrm{H}}\left(250 \mathrm{MHz} ; \mathrm{CDCl}_{3}\right) 0.45-1.92(16 \mathrm{H}, \mathrm{m})$, $0.81(3 \mathrm{H}, \mathrm{s}), 1.00(3 \mathrm{H}, \mathrm{s}), 1.94-2.42(6 \mathrm{H}, \mathrm{m}), 4.45$ (1H, app q, J, 7.9, CHO), $7.15-$ $7.35(10 \mathrm{H}, \mathrm{m}, \mathrm{ArCH}) ; \delta_{\mathrm{P}}\left(101 \mathrm{MHz} ; \mathrm{CDCl}_{3}\right)-11.92$.

\section{References}

1 Ligand 1: Andrey, O.; Camuzat-Dedenis, B.; Chabaud, L. Julienne, K.; Landais, Y.; Parra-Rapado, L.; Renaud, P. Tetrahedron 2003, 59, 8543-8550. Ligand 3: Bigdeli, M. A.; Nikje, M. M. A.; Heravi, M. M. Phosphorus, Sulfur Silicon Relat. Elem., 2002, 177, 2309-2314. Ligand 4: Altenhoff, G.; Goddard, 
R.; Lehmann, C. W.; Glorius, F. J. Am. Chem. Soc. 2004, 26, 15195-15201.

Ligand 5: Iwata, T.; Miyake, Y.; Nishibayashi, Y.; Uemura, S. J. Chem. Soc., Perkin Trans. 1, 2002, 1548-1554.

2

3

Jones, S.; Smanmoo, C. Tetrahedron Lett. 2004, 45, 1585-1588.

Bull, S. D.; Davies, S. G.; Jones, S.; Sanganee, H. J. J. Chem. Soc., Perkin Trans. 1, 1999, 387-398.

Russell, G. A.; N. Preecha; Yuh W. W. J. Am. Chem. Soc. 1989, 111, 49214927.

Guijarro, D.; Mancheño, B.; Yus, M. Tetrahedron 1994, 50, 8551-8558.

Zhang, H. X.; Guibe, F.; Balavoine, G. J. Org. Chem. 1990, 55, 1857-1867.

Jones, S.; Selitsianos, D.; Thompson, K. J.; Toms, S. M. J. Org. Chem. 2003, $68 ; 13,5211-5216$.

Schole, J.; Schole, C.; Eikemeyer, J.; Krebs, H. C. Tetrahedron 1994, 50, $1125-1128$.

Nitta, Y.; Arakawa, Y.; Ueyama, N. Chem. Pharm. Bull. 1986, 34, 27102718 .

Mark,V.; Van Wazer, J. R. J. Org. Chem. 1967; 32, 1187-1189.

Heinicke, J.; Boehle, I.; Tzschach, A.; J. Organomet. Chem. 1986, 317, 11-22.

Monnier, P. E.; Botella, J. M.; Murillo, A.; Klaebe, A.; Perie, J. Tetrahedron

1986, 42, 1315-1332.

Lavey, B. J.; Janda, J. D. J. Org. Chem. 1996, 61, 7633-7636.

Sosnovsky, G.; Zahret, E. H.; Schmidt, K. D. J. Org. Chem. 1970, 35, 336340.

Jones, S.; Selitsianos, D. Org. Lett. 2002, 4, 3671-3773.

Ba-Saif, S. A.; Waring, M. A.; Williams, A. J. Am. Chem. Soc. 1990, 112, 8185-8120.

Eguchi, C.; Suzuki, K.; Imahori, K. J. Biochem. 1977, 81, 1401-1411. 